\title{
Das pralle Leben
}

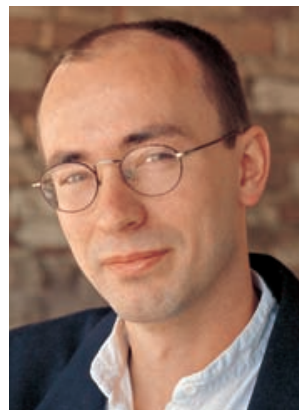

Dr. med. Dieter Schmid, Redaktionsleitung
Kleines Rätsel zur Begrüßung: Was ist das invasivste diagnostische Verfahren, das die Medizin zu bieten hat? Ein Herzkatheter? Die ERCP mit Schlitzung der Papilla vateri? Die Hirnbiopsie bei einem unklaren neurologischen Leiden? Völlig falsch! Ich spreche vom guten alten Hausbesuch. Mit dieser Maßnahme dringen Sie zwar nicht in den Körper des Patienten ein, dafür aber in sein Umfeld, seine Privatsphäre, seine Lebenswelt - kurz: das, was seine eigentliche Person ausmacht. Hier müssen Sie Risikofaktoren z. B. nicht erfragen, Sie können sie - zum Teil - mit allen Sinnen erkennen. Nun verliert ausgerechnet dieses zentrale ärztliche Instrument zusehends an Bedeutung. Die Zahl häuslicher Visiten ist in den Jahren nach 1996 um über 40\% zurückgegangen [1]. Die Gründe hierfür sind vielseitig. Die miese Bezahlung für diese Dienstleistung mag eine Rolle spielen. Anderen ist die ganze Aktion „Besuch“ schlicht zu aufwendig: hinfahren, Treppenhaus erklimmen, Schwätzchen halten, sich Probleme erzählen lassen ... Bleibt da überhaupt noch Zeit für Medizin? Die Hausärzte, mit denen wir für unseren Artikel „Weiterbildung Allgemeinmedizin“ (S. 22) gesprochen haben, haben da eine klare Meinung: Das IST Medizin!

Hierzulande mögen Hausbesuche eine oft unterschätzte Stütze des Gesundheitssystems sein. Woanders wäre ohne sie überhaupt keine Medizin möglich. Im Artikel „Im Schatten des Waldes“ (S. 14) erzählt der deutsche Mediziner Daniel Peter von seiner Arbeit als „Flussarzt“ bei den Urarina-Indianern am Rio Chambira im peruanischen Regenwald. Macht er sich auf einen „Hausbesuch“, ist er in seinem kleinen Boot Tage unterwegs. Den Weg zu seinen Patienten muss er sich mitunter mit Machete und Motorsäge freikämpfen. Die Urarina sind ein winziges Volk von 3.300 Seelen. Ohne die Visiten der europäischen Ärzte, die sie gegen von Holzfällern und Ölarbeitern eingeschleppte Infektionserreger impfen und eine Minimalversorgung gewährleisten, könnten sie nicht überleben.

Noch vor 150 Jahren war es hierzulande nicht anders. Da es noch keine guten Kliniken gab, wurden die meisten schwer kranken Patienten zu Hause behandelt. Bei Wind und Wetter eilten die Ärzte von Haus zu Haus. Dass sich dieser Aufwand reduziert hat, ist für die Mediziner natürlich ein Gewinn. Auf der anderen Seite: Nicht nur Patienten profitieren von Hausbesuchen. Auch Ärzten tut es gut, wenn sie ab und an die Weißkittelbrille abstreifen - und bei Hausbesuchen dem „prallen Leben“ ins Gesicht blicken: Sei es in Form der Blümchentapete der 90-jährigen Diabetikerin oder in Gestalt des Unrats in der Wohnung des 30-jährigen Messies. Solche Einblicke schützen sehr effektiv davor, sich zu sehr in einen Kokon aus Diagnose, Verordnungen und Prognose einzuspinnen ...

In diesem Sinne: Viel Spaß mit dem aktuellen Hausbesuch Ihrer Via medici wünscht Ihnen Ihr

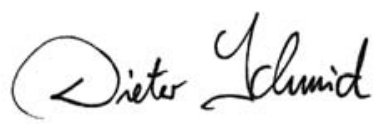

\section{ONLINE-UMFRAGE}

\section{Traumfach Allgemeinmedizin?}

Im Jahr 2020 werden in Deutschland etwa 15.000 Hausärzte fehlen. Viele junge Ärzte haben Angst vor einem Schreckensszenario, in dem sie rund um die Uhr für die Versorgung Hunderter Patienten zur Verfügung stehen müssen. Zudem ist ein Bild von hausärztlicher Medizin verbreitet, das sich auf banale Erkrankungen wie „Husten, Schnupfen, Heiserkeit“ reduziert. Die Ärzte, die wir für unseren „Weiterbildungsplaner Allgemeinmedizin“ interviewt haben, schwärmen dagegen vom großen medizinischen
Spektrum und den Vorteilen einer lebenslangen Begleitung ihrer Patienten. Für sie ist der Hausarzt der ideale Arzt, da er seine Patienten durch Sturm und Regen bis ans Sterbebett betreut. Welche Sichtweise bevorzugen Sie?

- Ist die Allgemeinmedizin eine berufliche Perspektive, die für Sie in Frage kommt?

Machen Sie mit und kommentieren Sie unter: www.thieme.de/viamedici/aktuelles/ aktion/miniumfrage1-11.html
- Ergebnis der Umfrage in Via medici 5.10: Wir wollten wissen, wie die seelische Befindlichkeit deutscher Medizinstudierender ist. Sie sollten sechs Fragen zu den Warnsignalen einer Depression durchlesen und angeben, wie oft Sie mit „Ja“ antworten mussten: 85\% der Teilnehmer bejahten mehr als eine Frage. Demnach wären die meisten Jungmediziner gefährdet, eine Depression zu entwickeln. Dagegen spricht das Deutsche Studentenwerk von nur etwa $8 \%$ psychisch kranker Studenten. Die Wahrheit dürfte wohl dazwischen liegen. Das Ergebnis im Detail unter: .../aktuelles/aktion/miniumfrage5-10.html 